\title{
Dynamic Cerebral Autoregulation in Embolic Stroke of Undetermined Source
}

\author{
Hongyin Ma', Jia Liu'2, Shan Lv', Peng Zhang', Wei-Tong Guo', Yang Qu', \\ Zhen-Ni Guo ${ }^{3 *}$ and Yi Yang ${ }^{1 *}$ \\ ${ }^{1}$ Stroke Center, Department of Neurology, The First Hospital of Jilin University, Changchun, China, ${ }^{2}$ Shenzhen Institutes \\ of Advanced Technology, Chinese Academy of Sciences, Shenzhen, China, ${ }^{3}$ Neuroscience Center, Department \\ of Neurology, The First Hospital of Jilin University, Changchun, China
}

\section{OPEN ACCESS}

Edited by:

Francesco Moccia,

University of Pavia, Italy

Reviewed by:

Lucy Beishon,

University of Leicester,

United Kingdom

Nils H. Petersen,

Yale University, United States

*Correspondence:

Yi Yang

doctoryangyi@163.com

Zhen-Ni Guo

zhen1ni2@163.com

Specialty section:

This article was submitted to

Vascular Physiology,

a section of the journal

Frontiers in Physiology

Received: 30 April 2020

Accepted: 28 September 2020

Published: 20 October 2020

Citation:

Ma H, Liu J, Lv S, Zhang P

Guo W-T, Qu Y, Guo Z-N and Yang Y

(2020) Dynamic Cerebral Autoregulation in Embolic Stroke

of Undetermined Source.

Front. Physiol. 11:557408.

doi: $10.3389 /$ fphys.2020.557408
Background and Purpose: Dynamic cerebral autoregulation (dCA) in acute ischemic stroke is probably compromised. Although the characteristics of dCA in different types of stroke have been largely investigated, dCA in embolic stroke of undetermined source (ESUS) remains poorly understood. In this group, we aimed to elucidate the characteristics of dCA and their relevance to clinical outcomes.

Methods: The study enrolled 77 ESUS patients and 50 controls. Bilateral cerebral blood flow velocities (CBFV) of middle cerebral arteries and arterial blood pressure were simultaneously recorded using a transcranial Doppler combined with a servo-controlled finger plethysmograph. Transfer function analysis was used to obtain dCA parameters including phase, gain, coherence at very low frequency (VLF) and low frequency (LF), and the rate of recovery (RoRc) of CBFV. A multivariable logistic regression model was established to explore the relationship between dCA and clinical outcomes.

Results: Gain at VLF and LF, phase at LF, and RoRc of CBFV in bilateral hemispheres of the ESUS group were consistently worse than those of the control group (all $P<0.001$ ). Bilateral RoRc of CBFV was significantly higher in patients with favorable outcomes than in those with unfavorable outcomes (stroke hemisphere: $P<0.001$; non-stroke hemisphere, $P=0.029$ ). Rate of recovery of CBFV in stroke hemisphere $>13.3 \% / \mathrm{s}$ was an independent predictor of favorable clinical outcomes (adjusted odds ratio $=30.95$, 95\% Cl: 5.33-179.81, $P<0.001)$.

Conclusions: Dynamic cerebral autoregulation was relatively impaired in both stroke and non-stroke hemispheres in ESUS patients, and functioning dCA after ESUS may indicate favorable clinical outcomes.

Keywords: cerebral autoregulation, vascular function, transcranial Doppler, transfer function analysis, ischemic stroke

\section{INTRODUCTION}

Cerebral autoregulation (CA) maintains cerebral blood flow $(\mathrm{CBF})$ at an approximately constant level within a certain range of arterial blood pressure (ABP) fluctuations (Lassen, 1959). The use of transcranial Doppler (TCD) in clinical practice has led to the gradual acceptance of dynamic cerebral autoregulation (dCA), which characterizes the temporal capacity of CBF recovery 
following a sudden change in ABP (Aaslid et al., 1989). As reduced effectiveness of autoregulation renders the brain more sensitive to both hypo- and hyperperfusion, dCA measurements are of significance in a variety of pathological settings, particularly in stroke patients. Dynamic cerebral autoregulation in acute ischemic stroke (AIS) patients is impaired to a certain degree, and the evidence accumulated over recent years suggests that dCA enables good prediction of clinical outcomes (Castro et al., 2017; Chi et al., 2018; Ma et al., 2018; Intharakham et al., 2019). Due to distinct pathological changes, the characteristics of dCA in different stroke subtypes may vary. Among all stroke subtypes, research into large artery atherosclerosis and small artery occlusion subtypes has been of substantial focus (Immink et al., 2005; Guo et al., 2014, 2015; Petersen et al., 2015). Nevertheless, the underlying pathogenesis of stroke with undetermined cause (cryptogenic stroke) has remained elusive, and dCA characteristics of this stroke subtype remain underexplored.

As a typical subset of cryptogenic stroke, embolic stroke of undetermined source (ESUS) has been proposed as a non-lacunar brain infarct without proximal arterial stenosis or cardioembolic sources. This concept was originally put forward in 2014 as a therapeutically relevant entity based on evidence that most cryptogenic strokes were embolic (Hart et al., 2014). In fact, ESUS patients comprise, on average, $17 \%$ of all ischemic strokes (Hart et al., 2017). To the best of our knowledge, the characteristics of dCA in cryptogenic stroke or ESUS remain underexplored. A preliminary dCA study of the cryptogenic stroke population indicated that dCA was compromised only in the non-affected hemisphere (Tutaj et al., 2014). Furthermore, although ESUS patients may be included in some dCA studies of AIS, this subgroup has never been specifically analyzed (Saeed et al., 2013; Llwyd et al., 2018; Ma et al., 2018). In our previous dCA study of AIS patients (Ma et al., 2018), eight ESUS patients were enrolled. Compared to healthy controls, ESUS patients tended to exhibit bilateral impairment of dCA, but the generalizability of these findings and specific autoregulatory characteristics require further confirmation. Extending our dCA observations of ESUS patients may contribute to deeper understanding of stroke pathogenesis and provide individualized information to optimize therapeutic strategies in the future.

Transfer function analysis (TFA) is one of the most popular approaches to quantify dCA. It models dCA as a linear control system, where ABP is considered as the input and cerebral blood flow velocity (CBFV) as the output of the system, which maintains $\mathrm{CBFV}$ at a relatively constant level despite changes in ABP. The autoregulatory parameters, including phase, gain, and coherence in the frequency domain, can be derived from TFA to characterize dCA. In particular, large phase indicates that CBFV does not follow the changes of ABP (normal dCA), whereas small phase suggests impaired dCA. Gain can be used to quantify the change in magnitudes. Coherence is a metric of linearity between $\mathrm{ABP}$ and $\mathrm{CBFV}$. When coherence is low, the assumption of linearity between $\mathrm{ABP}$ and $\mathrm{CBFV}$ is violated and the data needs to be discarded. In the time domain, the step response of $\mathrm{CBFV}$ indicates the recovery of $\mathrm{CBF}$ when a stepwise change in ABP occurs. A low rate of recovery (RoRc) of CBFV reflects a slow recovery of $\mathrm{CBFV}$, which also indicates that the autoregulation is impaired.

Therefore, this study aimed to elucidate the characteristics of dCA in ESUS patients and their relevance to clinical outcomes.

\section{MATERIALS AND METHODS}

\section{Patients and Controls}

This prospective observational study was performed at the Comprehensive Stroke Center, Department of Neurology, First Hospital of Jinlin University, China, from October 2017 to February 2019. This study complied with the Declaration of Helsinki, and ethical approval for the study was obtained from the Ethics Committee of the First Hospital of Jilin University, China (No. 2017-448). Written informed consent was obtained from all subjects or their direct relatives. Embolic stroke of undetermined source patients were diagnosed according to the criteria and protocol proposed by Hart et al. (2014). A series of diagnostic assessments were routinely arranged including brain computed tomography and brain magnetic resonance imaging, 12-lead electrocardiogram, Holter monitoring lasting for $24 \mathrm{~h}$, transthoracic echocardiography, and extracranial and intracranial vascular imaging evaluations (typically TCD, brain magnetic resonance angiography, and carotid ultrasound). In addition to ESUS diagnosis, patients were required to satisfy the following criteria: (1) admitted to hospital within 7 days after stroke onset; (2) brain infarction involved in anterior circulation territory; (3) modified Rankin Scale (mRS) score of 0 prior to stroke; (4) sufficient bilateral temporal bone window for TCD insonation; and (5) conscious and could fully cooperate with dCA measurement. Patients with (1) a history of stroke within 3 months, (2) more than 50\% stenosis or occlusion of intracranial and/or extracranial major artery in the non-stroke hemisphere, and (3) myocardial infarction, heart failure, severe anemia, and hyperthyroidism were excluded from the study.

In total, 50 age- and sex-matched volunteers without a history of stroke were enrolled as a control group. Control subjects underwent TCD and carotid ultrasound before dCA measurements to exclude asymptomatic intracranial and/or extracranial artery stenosis/occlusion.

\section{Clinical Data}

All patients received routine stroke treatment and intensive nursing care in the stroke unit by the same clinical team in accordance with early stroke management guidelines (Powers et al., 2018). Demographic information, disease history, and laboratory tests of risk factors for stroke were recorded. Neurological examination and National Institutes of Health Stroke Scale (NIHSS) scores were evaluated in a timely manner at admission and discharge for each patient. Neuroimaging information was scanned, and the characteristics of stroke lesions were classified manually by two senior neuroradiologists. Functional outcomes were evaluated using $\mathrm{mRS}$ at 3 months after 
stroke onset. Favorable outcomes were defined as mRS scores $<3$ at 3 months.

\section{DCA Measurements}

Dynamic cerebral autoregulation measurements were performed after the diagnosis of ESUS was established, and each patient was measured once during hospitalization, within 3-10 days after stroke onset. The measurements were performed in an exclusive laboratory room with minimal visual and acoustic stimulation. Room temperature was maintained at $22-24^{\circ} \mathrm{C}$. Subjects were instructed to refrain from alcohol and caffeine intake and engaging in excessive exercise for at least $12 \mathrm{~h}$ before measurements. All measurements were performed by one specialized neurovascular ultrasound doctor. As preparation before measurements, subjects were instructed to rest in a relaxed supine position for $10 \mathrm{~min}$. Baseline blood pressure was measured at the left brachial artery with an automatic blood pressure monitor (Omron 711, China). Bilateral middle cerebral arteries (MCA) were probed through temporal bone windows at a depth of 45-60 mm with 2-MHz probes applying TCD (MultiDop $\mathrm{X} 2$, DWL, Sipplingen, Germany) to record non-invasive CBFV. A servo-controlled plethysmograph (Finometer Pro, Netherlands) was used to continuously record spontaneous ABP. End tidal $\mathrm{CO}_{2}$ was measured using a capnograph with a face mask attached to the nasal cannula. After the $\mathrm{ABP}$ and $\mathrm{CBFV}$ signals were steadily obtained, real-time recording of measurements was initiated. During measurements, subjects were instructed to stay awake, breathe spontaneously, and minimize body movements for at least $5 \mathrm{~min}$. After measurements, the data were stored in a personal computer for subsequent dCA analysis.

\section{DCA Analysis}

Dynamic cerebral autoregulation analysis was performed using MATLAB (MathWorks, Natick, MA, United States). To eliminate possible time lags, beat-to-beat alignment of the data was achieved with a cross-correlation function. A third-order Butterworth low-pass filter (cutoff at $0.5 \mathrm{~Hz}$ ) was applied as an anti-alias filter before down-sampling the data to $1 \mathrm{~Hz}$. TFA was used to calculate the phase shift, gain, and coherence between ABP and CBFV at very low frequency (VLF, 0.02$0.07 \mathrm{~Hz}$ ) and low frequency (LF, 0.07-0.20 Hz) bands (Zhang et al., 1998; Panerai, 2008) in the frequency domain. A value of coherence above 0.34 is considered valid for TFA (Claassen et al., 2016). In the time domain, the step response of CBFV denotes how fast the recovery of $\mathrm{CBF}$ is when a stepwise change in ABP occurs. To quantify the speed of recovery, RoRc of CBFV was defined as $\Delta \mathrm{CBFV} / \Delta \mathrm{t} \times 100 \%$, with the first 3 seconds being used for the calculation. According to a previous study (Aaslid et al., 1989), the reason for choosing a short interval of the step response is to fit it in a regression line, so as to quantify the cerebral vascular resistance (CVR) with its slope. A short interval from 1 to $3.5 \mathrm{~s}$ was chosen by Aaslid et al. (1989), because the regression line was in an approximately linear fashion within this range. In our previous study, we found that the first 3 seconds of the step response can be well approximated by a regression line, while a longer interval of the step response can violate the assumption of linearity between $\mathrm{ABP}$ and $\mathrm{CBFV}$ when the step response starts to converge to a plateau (Ma et al., 2016). Therefore, we used the first 3 seconds of the step response (CBFV following a step change of $\mathrm{ABP}$ ) to calculate for the RoRc.

\section{Statistical Analyses}

All data were analyzed using Statistical Program for Social Sciences version 21.0 (SPSS, IBM, West Grove, PA, United States). For continuous variables, normality of data was determined using the Kolmogorov-Smirnov test. Normally distributed data are expressed as mean \pm SD, whereas nonnormally distributed data are expressed as median (interquartile range). Categorical variables are reported by the rate or constituent ratio. The Wilcoxon signed-rank test was used to compare the dCA parameters between stroke and nonstroke hemispheres. The Mann-Whitney U test was used to compare the dCA parameters between ESUS patients and controls. The values of the bilateral dCA parameters in the control group were all used for comparison regardless of hemisphere. The chi-squared test was used to compare the differences between categorical variables. Univariate analyses were applied to identify correlations between clinical factors and dCA parameters. For continuous or ranked variables, Pearson/Spearman correlation was used. For two or multiple categorical variables, Mann-Whitney $U$ test or Kruskal-Wallis $\mathrm{H}$ test was adopted for the univariate analyses. Repeatedmeasures ANOVA was used to compare the general trend of dCA parameter of stroke and non-stroke hemispheres between patients with favorable and unfavorable outcomes groups. A multivariable logistic regression model was established to identify the predictive value of the $\mathrm{dCA}$ parameter on clinical outcomes. Variables were selected according to clinical consideration and significant results of univariate analyses $(P<0.1)$ as reference. A receiver operating characteristic (ROC) curve was generated to determine the optimal cut-off point of dCA parameters associated with favorable/unfavorable clinical outcomes. Statistical tests were two-tailed. $P<0.05$ was considered statistically significant.

\section{RESULTS}

Measurements of 77 ESUS patients were performed with satisfactory signal quality a median of 6 days (range 310 days) after stroke onset. Measurements of 50 control subjects without a history of stroke were also collected. The demographic and clinical characteristics of subjects are presented in Table 1. There were no significant differences in age, sex, history of hypertension, diabetes mellitus, or current smoking status between ESUS patients and controls. Among ESUS patients, 12 had lesions in both left and right hemispheres; thus, there were 89 stroke and 65 non-stroke hemispheres in total. Physiological parameters during dCA measurements are summarized in Table 2. Excluding mean ABP, which was significantly higher in the ESUS group than in the control 
TABLE 1 | Demographic and clinical information of participants.

\begin{tabular}{|c|c|c|c|}
\hline & $\begin{array}{c}\text { ESUS } \\
(N=77)\end{array}$ & $\begin{array}{l}\text { Controls } \\
(N=50)\end{array}$ & $P$ values \\
\hline Age, years old & $56.2 \pm 10.3$ & $53.2 \pm 12.6$ & 0.153 \\
\hline Sex, male & $63(81.8)$ & $40(80.0)$ & 0.798 \\
\hline \multicolumn{4}{|l|}{ Race } \\
\hline Asian & $77(100)$ & $50(100)$ & - \\
\hline Current Smoking & $40(51.9)$ & $21(42.0)$ & 0.273 \\
\hline Hypertension & $39(50.6)$ & $20(40.0)$ & 0.240 \\
\hline Diabetes mellitus & $19(24.7)$ & $10(20.0)$ & 0.540 \\
\hline Previous symptomatic stroke & $13(16.9)$ & - & \\
\hline \multicolumn{4}{|l|}{ Stroke lesion characteristics } \\
\hline Lesion(s) on the left hemisphere & $29(37.7)$ & & \\
\hline Lesion(s) on the right hemisphere & $36(46.8)$ & & \\
\hline Lesion(s) on the both cerebral hemispheres & $12(15.6)$ & & \\
\hline Single acute lesion & $40(51.9)$ & & \\
\hline Multiple acute lesions & $37(48.1)$ & & \\
\hline Only cortical lesion(s) involvement & $9(11.7)$ & & \\
\hline Only subcortical lesion(s) involvement & $51(66.2)$ & & \\
\hline Both cortical and subcortical involvement & $17(22.1)$ & & \\
\hline Rt-PA thrombolysis & $49(63.6)$ & & \\
\hline NIHSS on admission & $6(4-9)$ & & \\
\hline NIHSS on discharge & $2(1-4)$ & & \\
\hline mRS at 3 months & $1(0-3)$ & & \\
\hline \multicolumn{4}{|l|}{ Laboratory results } \\
\hline LDL-C, mmol/L & $2.95 \pm 0.8$ & & \\
\hline $\mathrm{HbA} 1 \mathrm{c}, \%$ & $5.6(5.4-6.5)$ & & \\
\hline Homocysteine, $\mu \mathrm{mol} / \mathrm{L}$ & $\begin{array}{c}14.2 \\
(10.6-26.1)\end{array}$ & & \\
\hline Uric acid, mmol/L & $333.5 \pm 95.4$ & & \\
\hline
\end{tabular}

Data are expressed as mean $\pm S D$, median (interquartile range) or absolute number (percentage) where appropriate. Rt-PA, recombinant tissue plasminogen activator; NIHSS, National Institutes of Health Stroke Scale; mRS, modified Rankin Scale; LDL-C, low density lipoprotein cholesterol; HbA1C, glycosylated hemoglobin A (1C).

TABLE 2 | Physiological data during dCA measurements.

\begin{tabular}{lcc}
\hline & ESUS $(\mathbf{N}=\mathbf{7 7})$ & Controls $(\mathbf{N}=\mathbf{5 0})$ \\
\hline MAP, mmHg & $104.1 \pm 13.1^{*}$ & $89.2 \pm 10.0$ \\
HR, beats/min & $68.0(63.0-76.0)$ & $68.0(63.8-74.3)$ \\
Mean MCA CBFV, cm/s & & \\
Stroke hemispheres & $59.3(48.0-79.3)$ & $61.7(56.4-68.5)$ \\
Non-stroke hemispheres & $61.3(52.2-72.8)$ & \\
tCCO $_{2}, \mathrm{mmHg}$ & $38.1 \pm 1.4$ & $38.0 \pm 1.4$ \\
\hline
\end{tabular}

Data are expressed as mean $\pm S D$ or median (interquartile range) where appropriate. dCA, dynamic cerebral autoregulation; MAP, mean arterial blood pressure; HR, heart rate; MCA, middle cerebral artery; CBFV, cerebral blood flow velocity; $\mathrm{EtCO}_{2}$, end tidal $\mathrm{CO}_{2}$. ${ }^{*} \mathrm{P}<0.05$ compared with healthy controls.

group (104.1 \pm 13.1 vs. $89.2 \pm 10.0, P<0.001)$, other physiological parameters including heart rate, $\mathrm{CBFV}$ in both stroke and non-stroke hemispheres, and end tidal $\mathrm{CO}_{2}$ were not significantly different between ESUS and control groups. The main dCA parameters including phase, gain, and RoRc of CBFV of ESUS patients and controls are presented in Figure 1.

\section{DCA Parameters in ESUS Patients vs. Controls \\ Phase}

In the ESUS group, phase was not significantly different between stroke and non-stroke hemispheres at VLF and LF (VLF stroke vs. non-stroke hemispheres: 53.93 [31.96-74.26] vs. 50.69 [24.71-75.47], $P=0.31$; LF stroke vs. non-stroke hemispheres: 28.42 [16.04-35.55] vs. 25.19 [14.77-36.84], $P=0.37$ ). Compared to the control group (VLF: 56.77 [35.3171.32]; LF: 40.45 [32.03-50.07]), the ESUS group had a lower phase in both stroke and non-stroke hemispheres at LF (both $P<0.001)$. This tendency was not significantly different at VLF bilaterally (stroke hemispheres, $P=0.60$; non-stroke hemispheres, $P=0.27$ ).

\section{Gain}

Gain at VLF and LF did not significantly differ between stroke and non-stroke hemispheres in ESUS patients (VLF stroke vs. non-stroke hemispheres: 0.74 [0.57-0.98] vs. 0.77 [0.56-0.99], $P=0.95$; LF stroke vs. non-stroke hemispheres: $1.18[0.86-1.40]$ vs. $1.16[0.96-1.57], P=0.82)$. The value of gain in both stroke and non-stroke hemispheres was significantly higher in the ESUS group than in the control group (VLF: 0.53 [0.40-0.70]; LF: 0.98 [0.81-1.17], all $P<0.001$ ) at the corresponding frequency.

\section{RoRc of CBFV}

Rate of recovery of $\mathrm{CBFV}(\% / \mathrm{S})$ was obtained to quantify the efficiency of step response. Consistently, this parameter in ESUS patients did not differ between stroke and nonstroke hemispheres (stroke vs. non-stroke hemispheres: 15.71 [8.91-21.41] vs. 14.25 [9.09-20.17], $P=0.14$ ). The value of both hemispheres was significantly lower in the ESUS group than in the control group (20.91 [16.74-27.92], both $P<0.001$ ).

\section{Clinical Factors Associated With DCA Parameters}

Clinical factors of ESUS patients that correlated with dCA parameters were screened. Significant results are presented in Table 3. A small but consistent trend toward older age and poorer bilateral gain values at both VLF and LF was observed, although this did not reach statistical significance. Moreover, patients with a single stroke lesion tended to share better gain at LF than those with multiple stroke lesions. A higher uric acid level was associated with a poorer phase at LF bilaterally. Besides, sex, disease history, mean ABP, baseline NIHSS, whether patients underwent recombinant tissue plasminogen activator thrombolysis, stroke location, the time for dCA measurements, and other laboratory results were not correlated with any dCA parameter in both stroke and non-stroke hemispheres. In addition, there was no difference in dCA parameters between minor stroke (NIHSS $\leq 4)$ and moderate stroke (NIHSS > 4) patients (all $P>0.05$ ) and no significant difference was found in dCA parameters between earlier (3-6 days) and later (7-10 days) measurements (all $P>0.05$ ). 


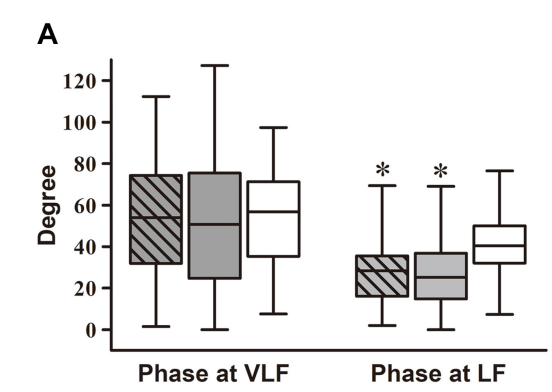

B

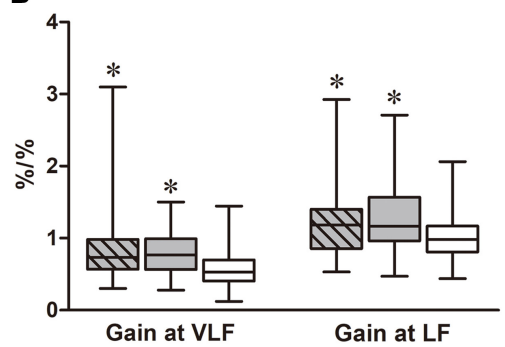

C

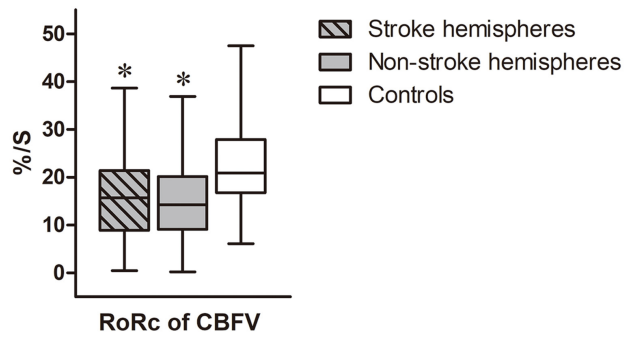

FIGURE 1 | Autoregulatory parameters in ESUS patients compared to controls. Box-and-whisker plots of (A) Phase of stroke and non-stroke hemispheres in ESUS patients and controls, at very low frequency (VLF, 0.02-0.07 Hz) and low frequency (LF, 0.07-0.20 Hz) bands, respectively. (B) Gain of stroke and non-stroke hemispheres in ESUS patients and controls, at VLF and LF bands, respectively. (C) The rate of recovery of cerebral blood flow velocity (RoRc of CBFV) of stroke and non-stroke hemispheres in ESUS patients and controls. "*" denotes $P<0.05$ for comparison with controls (at corresponding frequency bands).

\section{DCA Parameters for Predicting Functional Outcomes}

The median mRS score was 1 (interquartile range $0-3$ ) at 3 months. In the ESUS group, $26 \%$ of the patients exhibited unfavorable outcomes. There was no significant difference in the time for measurements between patients with favorable and unfavorable outcomes (favorable outcomes, 6 [5-7]; unfavorable outcomes, 6 [4-7]; $P=0.586)$. Bilateral RoRc of CBFV in patients with favorable outcomes was significantly higher than that of patients with unfavorable outcomes $(P=0.013)$, and there was no interaction between side and outcome $(P=0.10)$. For other dCA parameters, there were no significant differences between patients with favorable outcomes and unfavorable outcomes. According to the results of the Spearman correlation, a higher RoRc of CBFV in the stroke hemisphere was correlated with lower $\mathrm{mRS}(r=-0.246, P=0.031)$, indicating better functional outcomes, and a higher phase at VLF in the nonstroke hemisphere was correlated with lower mRS $(r=-0.254$, $P=0.041$ ) (Table 3); no other significant correlation was detected between dCA parameters and mRS. Besides the dCA parameters, NIHSS at admission $(r=0.292, P=0.010)$, NIHSS

TABLE 3 | Clinical factors of ESUS patients correlated with dCA parameters.

\begin{tabular}{lcccc}
\hline Clinical factors & $\begin{array}{c}\text { dCA } \\
\text { parameters }\end{array}$ & Hemisphere Coefficient & $\boldsymbol{P}$ Value \\
\hline Age & Gain at VLF & Stroke & 0.219 & 0.055 \\
Age & Gain at VLF & Non-stroke & 0.203 & 0.105 \\
Age & Gain at LF & Stroke & 0.196 & 0.087 \\
Age & Gain at LF & Non-stroke & 0.242 & 0.052 \\
Single/multiple stroke lesion(s) & Gain at LF & Stroke & - & 0.093 \\
Single/multiple stroke lesion(s) & Gain at LF & Non-stroke & - & $0.002^{*}$ \\
Uric acid & Phase at LF & Stroke & -0.318 & $0.005^{*}$ \\
Uric acid & Phase at LF & Non-stroke & -0.290 & $0.019^{*}$ \\
mRS at 3 months & Phase at VLF & Non-stroke & -0.254 & $0.041^{*}$ \\
mRS at 3 months & RoRc of CBFV & Stroke & -0.246 & $0.031^{*}$ \\
\hline
\end{tabular}

$d C A$, dynamic cerebral autoregulation; VLF, very low frequency; $L F$, low frequency; mRS, modified Rankin Scale; RoRc of CBFV, the rate of recovery of cerebral blood flow velocity. ${ }^{*} P<0.05$ in according statistical analyses. at discharge $(r=0.610, P<0.001)$, and uric acid level ( $r=-0.268, P=0.019$ ) were associated with clinical outcomes in the ESUS group. In the subsequent regression model for predicting clinical outcomes, the independent variables including age, sex, time for dCA measurements, NIHSS at admission, uric acid level, and RoRc of CBFV on stroke hemisphere were involved. The optimal cut-off value of RoRc of CBFV on stroke hemisphere for predicting clinical outcomes was $13.3 \% / \mathrm{s}$ (specificity of $77 \%$ and sensitivity of $75 \%$ ). The ROC curve analysis suggested that the area under the curve (AUC) was 0.77 (95\% CI: 0.66-0.88) (Figure 2). Rate of recovery of $\mathrm{CBFV}$ in the stroke hemisphere was dichotomized according to the optimal cut-off value. Multivariable logistic regression model revealed that RoRc of CBFV in the stroke hemisphere $>13.3 \% / \mathrm{s}$ was an independent predictor of favorable clinical outcomes (adjusted odds ratio $=30.95$, 95\% CI: $5.33-179.81$, $P<0.001)$.

\section{DISCUSSION}

To our knowledge, this study is the first clinical dCA assessment focusing on ESUS patients. Our findings suggested that dCA was likely impaired in both stroke and non-stroke hemispheres after stroke onset. Further, dCA may be a valuable clinical prognostic marker in ESUS patients.

Embolic stroke of undetermined source patients comprise one in six of all ischemic stroke patients, with a relatively younger age of onset and annual recurrence rates averaging $4.5 \%$ per year, despite milder stroke severity (Perera et al., 2016; Hart et al., 2017). However, the specific pathogenesis and various emboligenic mechanisms of this subgroup have not been fully clarified. The impact of CA on its pathogenesis and clinical outcomes remains unknown. We selected the ESUS population rather than cryptogenic stroke population as the ESUS population possesses a clearer definition and diagnostic protocol for screening to ensure accurate interpretation of results. In the pioneering work of Tutaj, six patients with AIS of undetermined etiology were enrolled, and the lower phase of non-affected hemispheres compared to that of controls indicated that dCA was compromised in the non-affected hemisphere 


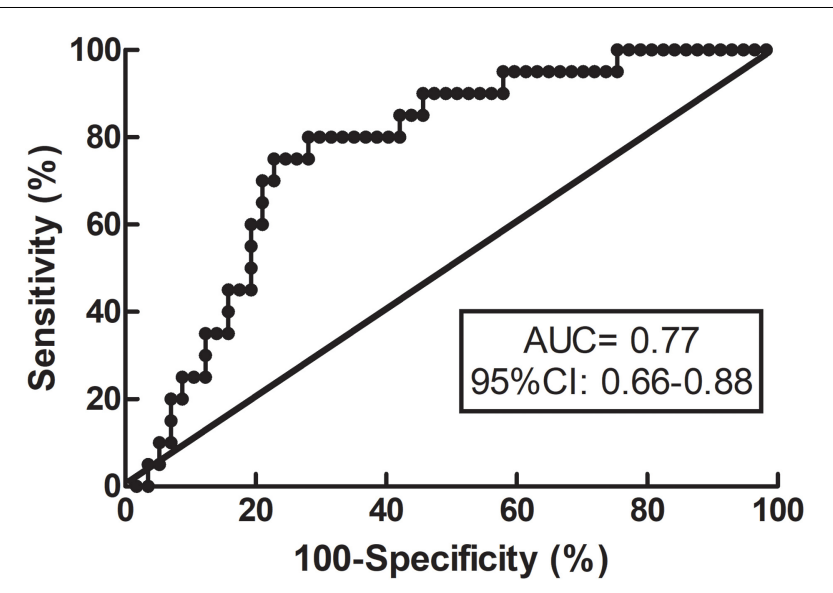

FIGURE 2 | Receiver operating characteristic (ROC) curve for RoRc of CBFV. $\mathrm{ROC}$ curve for RoRc of CBFV in the stroke hemisphere was generated to calculate the cut-off value that optimized the sensitivity and specificity for predicting functional outcomes. RoRc of CBFV, the rate of recovery of cerebral blood flow velocity.

but not in the affected hemisphere (Tutaj et al., 2014). In our previous work on AIS, patients were classified according to Trial of ORG 10172 in Acute Stroke Treatment (TOAST) criteria (Adams et al., 1993), and eight patients had cryptogenic stroke and concurrently satisfied the diagnosis of ESUS. We observed that the impairment in dCA tended to have bilateral involvement according to the lower phase in both affected and non-affected hemispheres based on detailed data, but the small sample size limited the generalizability of these findings. Thus, this current study enrolled more ESUS patients to clarify the dCA characteristics. Similar to previous observations, dCA was bilaterally impaired as verified by multiple dCA parameters, including phase, gain, and RoRc of CBFV. In this regard, changes in all parameters were not significantly different between hemispheres. To ensure the robustness of our results, controls comprising age- and sex-matched volunteers with comparable concomitant vascular risk factors were enrolled. Although we are unable to perform dCA measurements prior to stroke for comparison, we surmise that acute stroke lesions are unlikely to play a major role in impaired dCA for the following reasons. First, stroke volumes of ESUS patients were relatively small and occasionally scattered; these focal lesions may not directly lead to evident dysautoregulation and are even more unlikely to compromise the non-stroke hemisphere. Moreover, despite ruling out large artery arteriosclerosis and small artery occlusion for current stroke etiology, the basis of cerebral vasculopathy already existed prior to stroke owing to the cardiovascular risk factor burden (Perera et al., 2016), which is thought to undermine the structural and functional cerebral vasculature and give rise to impaired dCA. This has been demonstrated by numerous clinical imaging findings of macroangiopathy of the carotid artery and evidence of microangiopathy, such as white matter hyperintensity and cerebral microbleeds, which are frequently detected in the ESUS population (Coutinho et al., 2016; Kashima et al., 2018; Komatsu et al., 2018; Kikuno et al., 2020). In addition, direct proof of vascular endothelial function impairment in ESUS patients was identified in a recent study by Shirai using flow-mediated vasodilation tests, suggesting that endothelial function was impaired in ESUS patients compared to that in controls (Shirai et al., 2020). Therefore, impaired dCA in ESUS patients may have existed prior to stroke onset and exerted underlying detrimental effects on cerebral hemodynamics, rendering brain tissue more vulnerable to the burden of emboli and resulting in hypo-perfusion and irreversible infarction (Higuchi et al., 2020).

The evidence accumulated over recent years strongly suggests that bilaterally impaired dCA has been identified in both large and small artery stroke (Dawson et al., 2003; Saeed et al., 2013; Guo et al., 2014, 2015; Panerai et al., 2015; Xiong et al., 2017; Intharakham et al., 2019). The main difference lies in that the impairment is probably more evident in the stroke hemisphere of large artery stoke compared to that in the non-stroke hemisphere. By contrast, differences in dCA impairment were not significant between hemispheres for small artery stroke. Relative to the substantial information available on other subtypes, little is known regarding the ESUS population. Our findings on the ESUS population provide a comprehensive characterization of $\mathrm{dCA}$ in AIS. Collectively, our results suggest that although differences in pathogenesis and vasculopathy may exist in various subtypes, dCA in AIS may be bilaterally compromised when considering subtypes. Additionally, although multiple dCA parameters including gain at the VLF and LF bands, phase at the LF band, and the RoRc of CBFV in the bilateral hemispheres of the ESUS group were consistently worse than those of the control group, indicating bilaterally reduced damping effect, slower recovery, and less effectiveness of dCA in the ESUS population, it seemed that the RoRc of CBFV was the most relevant and stable parameter for predicting clinical outcomes according to later univariate and multivariate analyses, especially for the RoRc of CBFV in the stroke hemisphere, in which an optimal cut-off value could be generated for predicting clinical outcomes with a moderate specificity and sensitivity. These results of RoRc were not conflicting with other dCA parameters in terms of arriving at a conclusion of impaired autoregulation in ESUS consistently. Moreover, the results also suggested a superior predictive value of the RoRc among the dCA parameters in the ESUS patients. We speculate that the RoRc may detect a more rapid response of $\mathrm{CBFV}$ than phase and gain, as the RoRc is calculated from the slope of the first 3 seconds of the step response. This suggests that the RoRc represents a dynamic relationship at the high frequency $(\mathrm{HF}$ ) band (about $0.3 \mathrm{~Hz}$ ) which is different from the frequency ranges where phase (normally at the LF band) and gain (normally at the VLF band) are sensitive. In our future research, more attention will be paid on this parameter to further assess its potential clinical relevance and value. With respect to the effects of dCA for predicting the clinical outcomes, previous research has provided ample evidence. In Castro's study, dCA measurements were promptly performed within $6 \mathrm{~h}$ of ischemic stroke, and early effective autoregulation was proven to be associated with better neurological outcomes at 3 months (Castro et al., 2017). According to a recent study (Chi et al., 2018), mild AIS patients were enrolled for dCA assessments, and a predictive 
model was established. Their data revealed that phase $<61^{\circ}$ at VLF was independently associated with unfavorable outcomes. In agreement with these data, our previous observation of AIS patients indicated that the dCA parameters in the subacute stage were able to predict the clinical outcomes (Ma et al., 2018). Thus, combined with the results of our current study, dCA enabled good prediction of clinical outcomes applied to AIS and ESUS patients, and measuring dCA in clinical practice may be valuable for evaluating and establishing individualized therapeutic strategies.

This study has several limitations. First, due to restrictions of the TCD methodology, patients with poor temporal window penetration could not be measured successfully, which likely occurred in elderly female patients. Therefore, the composition of our study differed from the actual ESUS population, in which the proportion of men exceeds that of women; this may have generated a selection bias. Besides, given that cooperative and conscious patients were enrolled, the study more likely involved patients with milder stroke, which is another selection bias; thus, our findings cannot be generalized to severe stroke patients. Second, although we selected patients with stroke lesions involving the anterior circulation, some lesions may have involved the posterior circulation. The blood vessels for TCD monitoring are the bilateral MCA reflecting blood flow changes within the area of arterial blood supply; thus, dCA of the posterior circulation was not distinctively focused on in this study. Third, as dCA measurements were performed only once during the hospitalization within a wide range of 3-10 days after stroke onset, our data may not provide the characteristics of the temporal changes in dCA. Fourth, the optimal cut-off point was determined by generating an ROC curve and, then, dCA data were divided into two categories for further logistics regression analysis. This pre-specified cut-off value may lead to bias and should be considered when interpreting the data. Fifth, it should be noted that TCD was used to measure CBFV as a surrogate for CBF; thus, our results were based on the assumption that the vessel diameter of MCA remained constant over small physiological fluctuations, such as end tidal $\mathrm{CO}_{2}$ and $\mathrm{ABP}$.

\section{REFERENCES}

Aaslid, R., Lindegaard, K. F., Sorteberg, W., and Nornes, H. (1989). Cerebral autoregulation dynamics in humans. Stroke 20, 45-52. doi: 10.1161/01.str. 20.1.45

Adams, H.P. Jr, Bendixen, B. H., Kappelle, L. J., Biller, J., Love, B. B., Gordon, D. L., et al. (1993). Classification of subtype of acute ischemic stroke. Definitions for use in a multicenter clinical trial. TOAST. Trial of Org 10172 in Acute Stroke Treatment. Stroke 24, 35-41. doi: 10.1161/01.str.24.1.35

Castro, P., Serrador, J. M., Rocha, I., Sorond, F., and Azevedo, E. (2017). Efficacy of cerebral autoregulation in early ischemic stroke predicts smaller infarcts and better outcome. Front. Neurol. 8:113. doi: 10.3389/fneur.2017.00113

Chi, N.-F., Hu, H.-H., Wang, C.-Y., Chan, L., Peng, C.-K., Novak, V., et al. (2018). Dynamic cerebral autoregulation is an independent functional outcome predictor of mild acute ischemic stroke. Stroke 49, 2605-2611. doi: 10.1161/ STROKEAHA.118.022481

Claassen, J. A. H. R., Meel-Van Den Abeelen, A. S. S., Simpson, D. M., Panerai, R. B., and International Cerebral Autoregulation Research Network. (2016). Transfer function analysis of dynamic cerebral autoregulation: a white paper from the

\section{CONCLUSION}

In summary, dCA of ESUS patients was relatively impaired in both stroke and non-stroke hemispheres. Dynamic cerebral autoregulation may be a clinical prognostic marker in ESUS patients. As such, dCA measurements in clinical practice may be a useful research tool as well as a routine evaluation method for ESUS and other stroke patients.

\section{DATA AVAILABILITY STATEMENT}

The raw data supporting the conclusions of this article will be made available by the authors, without undue reservation.

\section{ETHICS STATEMENT}

The studies involving human participants were reviewed and approved by the Ethics Committee of The First Hospital of Jilin University. The patients/participants provided their written informed consent to participate in this study.

\section{AUTHOR CONTRIBUTIONS}

HM, Z-NG, and YY designed the study. SL, W-TG, and YQ performed the data collection. HM, JL, and PZ performed the analyses. HM wrote the manuscript. All authors interpreted the data and had full access to the data and helped critically revise the manuscript before reviewing and approving the final version.

\section{FUNDING}

This project was supported by the National Key R\&D Program of China (2016YFC1301600), Program for Jilin University Science and Technology Innovative Research Team (2017TD-12), and Jilin Provincial Key Laboratory (20190901005JC) to YY.

International Cerebral Autoregulation Research Network. J. Cereb. Blood Flow Metab. 36, 665-680. doi: 10.1177/0271678X15626425

Coutinho, J. M., Derkatch, S., Potvin, A. R. J., Tomlinson, G., Kiehl, T.-R., Silver, F. L., et al. (2016). Nonstenotic carotid plaque on CT angiography in patients with cryptogenic stroke. Neurology 87, 665-672. doi: 10.1212/WNL. 0000000000002978

Dawson, S. L., Panerai, R. B., and Potter, J. F. (2003). Serial changes in static and dynamic cerebral autoregulation after acute ischaemic stroke. Cerebrovasc. Dis 16, 69-75. doi: 10.1159/000070118

Guo, Z. N., Liu, J., Xing, Y., Yan, S., Lv, C., Jin, H., et al. (2014). Dynamic cerebral autoregulation is heterogeneous in different subtypes of acute ischemic stroke. PLoS One 9:e93213. doi: 10.1371/journal.pone. 0093213

Guo, Z. N., Xing, Y., Wang, S., Ma, H., Liu, J., and Yang, Y. (2015). Characteristics of dynamic cerebral autoregulation in cerebral small vessel disease: diffuse and sustained. Sci. Rep. 5:15269. doi: 10.1038/srep15269

Hart, R. G., Catanese, L., Perera, K. S., Ntaios, G., and Connolly, S. J. (2017). Embolic stroke of undetermined source: a systematic review and clinical update. Stroke 48, 867-872. doi: 10.1161/STROKEAHA.116.016414 
Hart, R. G., Diener, H. C., Coutts, S. B., Easton, J. D., Granger, C. B., O'donnell, M. J., et al. (2014). Embolic strokes of undetermined source: the case for a new clinical construct. Lancet Neurol. 13, 429-438. doi: 10.1016/s1474-4422(13) 70310-7

Higuchi, E., Toi, S., Shirai, Y., Hoshino, T., Ishizuka, K., Shimizu, S., et al. (2020). Prevalence of microembolic signals in embolic stroke of undetermined source and other subtypes of ischemic stroke. Stroke 51, 655-658. doi: 10.1161/ STROKEAHA.119.027008

Immink, R. V., Van Montfrans, G. A., Stam, J., Karemaker, J. M., Diamant, M., and Van Lieshout, J. J. (2005). Dynamic cerebral autoregulation in acute lacunar and middle cerebral artery territory ischemic stroke. Stroke 36, 2595-2600. doi: 10.1161/01.str.0000189624.06836.03

Intharakham, K., Beishon, L., Panerai, R. B., Haunton, V. J., and Robinson, T. G. (2019). Assessment of cerebral autoregulation in stroke: a systematic review and meta-analysis of studies at rest. J. Cereb. Blood Flow Metab. 39, 2105-2116. doi: 10.1177/0271678X19871013

Kashima, S., Shimizu, T., Akiyama, H., and Hasegawa, Y. (2018). Magnetic resonance imaging white matter hyperintensity as a predictor of stroke recurrence in patients with embolic stroke of undetermined source. J. Stroke Cerebrovasc. Dis. 27, 3613-3620. doi: 10.1016/j.jstrokecerebrovasdis.2018. 08.033

Kikuno, M., Ueno, Y., Shimizu, T., Kuriki, A., Tateishi, Y., Doijiri, R., et al. (2020) Underlying embolic and pathologic differentiation by cerebral microbleeds in cryptogenic stroke. J. Neurol. 267, 1482-1490. doi: 10.1007/s00415-0002009732-00414

Komatsu, T., Iguchi, Y., Arai, A., Sakuta, K., Sakai, K., Terasawa, Y., et al. (2018). Large but Nonstenotic Carotid Artery Plaque in Patients With a History of Embolic Stroke of Undetermined Source. Stroke 49, 3054-3056. doi: 10.1161/ STROKEAHA.118.022986

Lassen, N. A. (1959). Cerebral blood flow and oxygen consumption in man. Physiol. Rev. 39, 183-238. doi: 10.1152/physrev.1959.39.2.183

Llwyd, O., Salinet, A. S. M., Panerai, R. B., Lam, M. Y., Saeed, N. P., Brodie, F., et al. (2018). cerebral haemodynamics following acute ischaemic stroke: effects of stroke severity and stroke subtype. Cerebrovascu. Dis. Extra 8, 80-89. doi: 10.1159/000487514

Ma, H., Guo, Z. N., Jin, H., Yan, X., Liu, J., Lv, S., et al. (2018). Preliminary study of dynamic cerebral autoregulation in acute ischemic stroke: association with clinical factors. Front. Neurol. 9:1006. doi: 10.3389/fneur.2018.01006

Ma, H., Guo, Z. N., Liu, J., Xing, Y., Zhao, R., and Yang, Y. (2016). Temporal course of dynamic cerebral autoregulation in patients with intracerebral hemorrhage. Stroke 47, 674-681. doi: 10.1161/strokeaha.115.011453

Panerai, R. B. (2008). Cerebral autoregulation: from models to clinical applications. Cardiovascu. Eng. 8, 42-59. doi: 10.1007/s10558-007-9044-6

Panerai, R. B., Jara, J. L., Saeed, N. P., Horsfield, M. A., and Robinson, T. G. (2015). Dynamic cerebral autoregulation following acute ischaemic stroke: comparison of transcranial Doppler and magnetic resonance imaging techniques. J. Cereb. Blood Flow Metab. 36, 2194-2202. doi: 10.1177/0271678x15615874

Perera, K. S., Vanassche, T., Bosch, J., Giruparajah, M., Swaminathan, B., Mattina, K. R., et al. (2016). Embolic strokes of undetermined source: prevalence and patient features in the ESUS Global Registry. Int. J. Stroke 11, 526-533. doi: $10.1177 / 1747493016641967$

Petersen, N. H., Ortega-Gutierrez, S., Reccius, A., Masurkar, A., Huang, A., and Marshall, R. S. (2015). Dynamic cerebral autoregulation is transiently impaired for one week after large-vessel acute ischemic stroke. Cerebrovasc. Dis. 39, 144-150. doi: 10.1159/000368595

Powers, W. J., Rabinstein, A. A., Ackerson, T., Adeoye, O. M., Bambakidis, N. C., Becker, K., et al. (2018). 2018 guidelines for the early management of patients with acute ischemic stroke: a guideline for healthcare professionals from the american heart association/american stroke association. Stroke 49, e46-e110. doi: 10.1161/STR.0000000000000158

Saeed, N. P., Panerai, R. B., Horsfield, M. A., and Robinson, T. G. (2013). Does stroke subtype and measurement technique influence estimation of cerebral autoregulation in acute ischaemic stroke? Cerebrovasc. Dis. 35, 257-261. doi: $10.1159 / 000347075$

Shirai, Y., Toi, S., Adachi, U., and Kitagawa, K. (2020). Impaired endothelial function in embolic stroke of undetermined source. J. Stroke Cerebrovascu. Dis. 29, 104489-104489. doi: 10.1016/j.jstrokecerebrovasdis.2019. 104489

Tutaj, M., Miller, M., Krakowska-Stasiak, M., Piştek, A., Hebda, J., Lạtka, M., et al. (2014). Dynamic cerebral autoregulation is compromised in ischaemic stroke of undetermined aetiology only in the non-affected hemisphere. Neurol. Neurochir. Pol. 48, 91-97. doi: 10.1016/j.pjnns.2013.12.006

Xiong, L., Tian, G., Lin, W., Wang, W., Wang, L., Leung, T., et al. (2017). Is dynamic cerebral autoregulation bilaterally impaired after unilateral acute ischemic stroke? J. Stroke Cerebrovasc. Dis. 26, 1081-1087. doi: 10.1016/j. jstrokecerebrovasdis.2016.12.024

Zhang, R., Zuckerman, J. H., Giller, C. A., and Levine, B. D. (1998). Transfer function analysis of dynamic cerebral autoregulation in humans. Am. J. Physiol. 274, $\mathrm{H} 233-\mathrm{H} 241$.

Conflict of Interest: The authors declare that the research was conducted in the absence of any commercial or financial relationships that could be construed as a potential conflict of interest.

Copyright (c) $2020 \mathrm{Ma}$, Liu, Lv, Zhang, Guo, Qu, Guo and Yang. This is an open-access article distributed under the terms of the Creative Commons Attribution License (CC BY). The use, distribution or reproduction in other forums is permitted, provided the original author(s) and the copyright owner(s) are credited and that the original publication in this journal is cited, in accordance with accepted academic practice. No use, distribution or reproduction is permitted which does not comply with these terms. 